\title{
Natural product cycloastragenol: a promising anti-diabetes candidate with therapeutic effects on its related complications in
} rats

Qi Zhang ${ }^{1}$, Sui-qi Cheng ${ }^{2}$, Jia-wei Sun², Zhi-yin Sun², Wang Peng ${ }^{2}$, Guo-tao Kuang ${ }^{2}$, Xiao-xuan $\mathrm{Su}^{2}$, Xiao-na $\mathrm{Wang}^{2}$, Yong $\mathrm{Rao}^{2}$, and Tian-miao $\mathrm{Ou}^{2}$

${ }^{1}$ Guangzhou University of Chinese Medicine

${ }^{2}$ Sun Yat-Sen University

April 28, 2020

\begin{abstract}
BACKGROUND AND PURPOSE Type II Diabetes mellitus (T2DM) is a worrying chronic metabolic disorder accompanied by multiple serious complications, makes threaten public health. In the present study, we evaluated the therapeutic effects against T2DM of the cycloastragenol (CAG), a key metabolite of astragaloside IV (AST) isolated from traditional Chinese plant Astragalus membranaceus and revealed its molecular mechanism. EXPERIMENTAL APPROACH The plasma glucosedecreasing effects of AST and CAG were monitored in KunMing mice by performing an OGTT test. Furthermore, the effect of CAG on the metabolism of Zuker diabetic fat (ZDF) rats was demonstrated after treatment for 5 weeks, as well as on diabetesrelevant clinical symptoms and glucose absorbance. The underlying molecular mechanism of the therapeutic effects of CAG was explored in both wild-type and sodium/glucose co-transporter 2 (SGLT2)-overexpressed HEK293 cells. KEY RESULTS CAG showed stronger effects in lowering plasma glucose and enhancing glucose tolerance than AST in KM mice and ZDF rats. In the ZDF rats, the potential antidiabetic properties of CAG were associated with amelioration in hyperglycemia, dyslipidemia, myocardial and kidney fibrosis. Interestingly, glucose reuptake was inhibited in the kidneys of mice treated with CAG, while urine glucose and sodium levels were elevated. The underlying mechanism might be that CAG reduced the expression of SGLT2 in the kidney and inhibited glucose transport as well. CONCLUSIONS AND IMPLICATIONS CAG can ameliorate the T2DMrelated metabolic syndromes of ZDF rats by acting on the SGLT2, which provides a certain references for the application of CAG in T2DM therapy.
\end{abstract}

Natural product cycloastragenol: a promising anti-diabetes candidate with therapeutic effects on its related complications in rats

Running title: A potential SGLT2 inhibitor from natural products

Qi Zhang ${ }^{\mathrm{b}}$, Suiqi Cheng ${ }^{\mathrm{a}}$, Jiawei Sun ${ }^{\mathrm{a}}$, Zhiyin $\mathrm{Sun}^{\mathrm{a}}$, Wang Peng ${ }^{\mathrm{a}}$, Guotao Kuang ${ }^{\mathrm{a}}$, Xiaoxuan $\mathrm{Su}^{\mathrm{a}}$, Xiaona Wang $^{\mathrm{a}}$, Yong Rao ${ }^{\mathrm{a}, *}$, Tianmiao $\mathrm{Ou}^{\mathrm{a}, *}$

${ }^{a}$ Guangdong Provincial Key Laboratory of New Drug Design and Evaluation, School of Pharmaceutical Sciences, Sun Yat-sen University, Guangzhou 510006, China

${ }^{b}$ School of Chinese Meteria Medica, Guangzhou University of Chinese Medicine, 510006, Guangzhou, China

* Corresponding Authors

Professor Tian-miao Ou, PhD

School of Pharmaceutical Sciences, Sun Yat-sen University 
Guangzhou, China

Tel: +862039943055

Fax: +862039943055

Email: outianm@mail.sysu.edu.cn

Yong Rao, PhD

School of Pharmaceutical Sciences, Sun Yat-sen University

Guangzhou, China

Tel: $+86-20-39943068$

Fax: + 86-20-39943056

Email: raoyong0805@126.com

\section{Bullet point summary}

What is already known

- Astragaloside IV (AST ) has been reported with the anti-hyperglycemia activity in mice.

- SGLT2 protein is a promising target for treating of T2DM

What this study adds

- The metabolite of AST , CAG , is more efficiently in combating T2DM.

- CAG inhibits glucose absorbance and promotes glucose excretion.

- CAG works mainly through the SGLT2.

\section{What is the clinical significance}

CAG should be considered as a new-type SGLT2 inhibitor for treating of T2DM.

\section{ABSTRACT}

\section{BACKGROUND AND PURPOSE}

Type II Diabetes mellitus (T2DM) is a worrying chronic metabolic disorder accompanied by multiple serious complications, makes threaten public health. In the present study, we evaluated the therapeutic effects against T2DM of the cycloastragenol (CAG ), a key metabolite of astragaloside IV (AST ) isolated from traditional Chinese plant Astragalus membranaceus and revealed its molecular mechanism.

\section{EXPERIMENTAL APPROACH}

The plasma glucose-decreasing effects of AST and CAGwere monitored in KunMing mice by performing an OGTT test. Furthermore, the effect of CAG on the metabolism of Zuker diabetic fat (ZDF) rats was demonstrated after treatment for 5 weeks, as well as on diabetes-relevant clinical symptoms and glucose absorbance. The underlying molecular mechanism of the therapeutic effects of CAG was explored in both wild-type and sodium/glucose co-transporter 2 (SGLT2)-overexpressed HEK293 cells.

\section{KEY RESULTS}

CAG showed stronger effects in lowering plasma glucose and enhancing glucose tolerance than AST in KM mice and ZDF rats. In the ZDF rats, the potential antidiabetic properties of CAGwere associated with amelioration in hyperglycemia, dyslipidemia, myocardial and kidney fibrosis. Interestingly, glucose reuptake was inhibited in the kidneys of mice treated with CAG, while urine glucose and sodium levels were elevated. The underlying mechanism might be that CAG reduced the expression of SGLT2 in the kidney and inhibited glucose transport as well. 


\section{CONCLUSIONS AND IMPLICATIONS}

CAG can ameliorate the T2DM-related metabolic syndromes of ZDF rats by acting on the SGLT2, which provides a certain references for the application of CAG in T2DM therapy.

Keywords: type 2 diabetes mellitus, Cycloastragenol, Astragaloside IV, hyperglycemia, cardiovascular disease, SGLT2.

\section{Abbreviations}

T2DM, Type II Diabetes mellitus; CAG, cycloastragenol; AST, astragaloside IV; SGLTs, sodium-glucose cotransporters; ZDF rats, Zuker diabetic fatty rat; FDA, Food and Drug Administration; SLCs, solute carriers; 2NBDG, 2-( $N$-(7-nitrobenz-2-oxa-1,3-diazol-4-yl) amino)-2-deoxyglucose; OGTT, oral glucose tolerance test; H\&E, hematoxylin and eosin; PAS, periodicacid-schiff stain; MetS, metabolic syndromes; HbA1c, glycated hemoglobin; LDL-c, low-density lipoprotein cholesterol; 14C-AMG, [14C]methyl-alphaD-glucopyranoside, TGF- $\beta 1$, transforming growth factor beta-1.

\section{Introduction}

Type II diabetes mellitus (T2DM) has become a global public health crisis, which poses a great threat to the health and quality of life of people in all countries, especially in developing countries (Moucheraud et al., 2019). T2DM is a chronic metabolic disease characterized by hyperglycemia and insulin resistance and accompanied by a series of clinical syndromes, including diabetic nephropathy (Tao et al., 2019), diabetic retinopathy (Dow et al., 2018; Ren et al., 2019), and cardiovascular complications (Vaidya et al., 2015). The FDA has approved several new drugs for T2DM, such as metformin (Hostalek et al., 2015; Pernicova \& Korbonits, 2014), rosiglitazone (Han et al., 2016) and Dapagliflozin (Scheen, 2017; Vivian, 2015). Unfortunately, although the use of these drugs can lower blood glucose to nearly normal levels, mortality from cardiovascular disease in diabetes either increased or was not affected (Gerstein et al., 2008; Navarro-Perez et al., 2018). Given this, the discovery of new drugs with new targets or specific molecular mechanisms is still of great concern.

Sodium-dependent glucose co-transporters (SGLTs) are solute carriers (SLCs) responsible for glucose (re)absorption (Rives et al., 2017; Wright et al., 2011). SGLTs are encoded by the SLC5A genes, contains multiple isoforms, mostly, SGLT1 and SGLT2. SGLT1 and SGLT2 are expressed in the kidney, and SGLT1 expressed in the small intestine as well (Rieg \& Vallon, 2018; Wood \& Trayhurn, 2003). Among them, SGLT2 is a high capacity transporter responsible for $90 \%$ of glucose reuptake and tissue-specific expressed in the renal tubules of the kidney (Abdul-Ghani et al., 2012; Wang et al., 2017). SGLT2 is a promising target for the treatment of diabetes due to the inhibition on it can reduce the risk of hyperglycemia by decreasing glucose reuptake (Chao \& Henry, 2010). Nowadays, eight SGLT2 inhibitors have been approved by the FDA for treating diabetes; they can increase urinary glucose excretion and display anti-hyperglycaemic effects that are primarily independent of insulin (Filippas-Ntekouan et al., 2018; Nauck, 2014; Shyangdan et al., 2016). Moreover, the efficacy and safety of SGLT2 inhibitors, especially in the prevention of cardiovascular diseases, have attracted full attention (Vaduganathan \& Butler, 2019). Nevertheless, the SGLT2 inhibitors on the market now are all glycoside compounds, and it is of considerable significance to further study whether we can expand new structure types in this field. For example, finding a novel structure from natural compounds.

Astragalus Membranaceus is used as a traditional Chinese medicine with multiple bioactivities, such as antihyperglycemia (Liu et al., 2019), anti-inflammation (Zhou et al., 2017), anti-fibrosis (Shan et al., 2016), and remission of diabetic nephropathy (Liu et al., 2017). Several key ingredients were identified from Astragalus Membranaceus, such as astragaloside IV (AST ), a soluble lanolin alcohol-shaped tetracyclic triterpenoid saponin. AlthoughAST shows a potential for treating T2DM (Zhang et al., 2020), it possesses low bioavailability (Cheng \& Wei, 2014; Ran et al., 2016). Instead, its aglycone cycloastragalol (CAG ) exhibits good bioavailability sinceCAG is the primary metabolite of AST in vivovia intestinal bacterial biotransformation (Szabo, 2014; Zhou et al., 2012). However, the effects of CAG on treating diabetes mellitus has not been reported so far. Therefore, the first purpose in the present study is to evaluate the 
therapeutic effect of CAG on T2DM from the perspective of the pathological feature by using the ZDF diabetic rat model. The second goal was to investigate the molecular patterns of CAG in the performance of these effects. These studies will provide some new ideas for the discovery of new anti-T2DM drugs with SGLT2 inhibitory activity.

\section{Materials and Methods}

\section{Materials}

All the compounds used in the present study were commercially available, including 2-(N-(7-Nitrobenz-2oxa-1,3-diazol-4-yl)Amino)-2-Deoxyglucose (2-NBDG, Invitrogen, USA, CID: 6711157), 2-deoxy-D-glocose (2DG, Invitrogen, USA, CID: 5287471), cytochalasin B (Shanghai Yuanye Bio-Technology Co., Ltd, China, CID: 5311281), metformin (Shanghai Yuanye Bio-Technology Co., Ltd, China, CID: 4091), Dapagliflozin (Shanghai Yuanye Bio-Technology Co., Ltd, China, CID: 9887712), , astragaloside IV (AST, Chengdu Mansit Bio-technology Co, Ltd, China, CID: 13943297), and cycloastragalol (CAG, Chengdu Mansit Bio-technology Co, Ltd, China, CID: 13943286). All detection kits were purchased from Nanjing Jiancheng Bioengineering Institute (Nanjing, China), and ELISA kits were obtained from Mlbio (USA). All other chemicals were of analytical grade and were obtained from Sigma (USA) and Merck (DE).

\section{Animal experiments}

Male KM mice (7-8 weeks, 18-22 g, RRID:MGI:5651867) were purchased from the Laboratory Animal Centre of Sun Yat-sen University (Guangzhou, China) and male Zucker Diabetic Fatty (ZDF) rats including ZDFLeprfa/fa rats and ZDF-Leprfa/ctrl rats (7-8 weeks, 220 240 g, RRID:RGD_2314027, RRID:RGD_2311071) were purchased from Beijing Vital River Laboratory Animal Technology (Beijing, China). All animal procedures were approved by the Sun Yat-sen University Committee on Ethics for the Use of Laboratory Animals in accordance with the Animal Welfare Legislation of China. Animal studies are reported in compliance with the ARRIVE guidelines.

$\mathrm{KM}$ mice were kept at $22 \pm 1{ }^{\circ} \mathrm{C}$ on a 12-hour light/dark cycle with free access to food and water. These mice were randomly divided into 6 groups, including lean group (without glucose administrated), vehicle group, metformin group, Dapagliflozin group, AST group andCAG group, each group included 10 mice. Metformin $(100 \mathrm{mg} / \mathrm{kg})$, Dapagliflozin $(1 \mathrm{mg} / \mathrm{kg})$, AST $(5 \mathrm{mg} / \mathrm{kg})$, CAG $(5 \mathrm{mg} / \mathrm{kg})$ were dissolved in normal saline containing 1\% CMC-Na and taken gavage administration in mice. The lean and the vehicle groups were administered the same volume. After acclimatization, oral glucose tolerance test (OGTT) was performed. Briefly, mice were fasted for a period of $14 \mathrm{~h}$ and then administrated with glucose $(2 \mathrm{~g} / \mathrm{kg})$ orally. The plasma glucose of $0,15,30,60$, and 120 min were detected by using a blood glucose meter (Roche), respectively.

ZDF-Lepr ${ }^{\text {fa/ctrl }}$ rats were fed a chow diet provided by the Animal Center of Sun Yat-sen University and ZDFLepr $^{\text {fa/fa }}$ rats were fed high fat diet 5008 (Labdiet, USA) for one month. Randomly selected the rats with blood glucose over $11.1 \mathrm{mmol} / \mathrm{L}$ from ZDF-Lepr ${ }^{\mathrm{fa}} / \mathrm{fa}$ rats before experiments. These ZDF rats were divided into 5 subgroups: lean group (ZDF-Lepr ${ }^{\mathrm{fa} / \mathrm{ctrl}}$, saline), vehicle group (ZDF-Lepr ${ }^{\mathrm{fa} / \mathrm{fa}}$, model), Dapagliflozin (1 $\mathrm{mg} / \mathrm{kg} / \mathrm{d}$, p.o. $)$, CAG (1 and $5 \mathrm{mg} / \mathrm{kg} / \mathrm{d}$, p.o. ). The OGTT experiment was preceded after the administration of CAG as described above. The urinary glucose, urinary sodium, and urine efflux were determined after 1or 5 -week treatment, respectively.

\section{Plasma analysis}

At the end of the study, fasted mice for eight hours and injected of ketamine/xylazine (i.p. ). After the mice were fully anesthetized, collected the whole blood from abdominal aorta in a tube containing $1 \mathrm{mM}$ EDTA for the measurement of relevant plasma parameters. Killed the mice by cervical decapitation. Weighed the tissues of interest and freeze-clamped or fixed them in a $4 \%$ formaldehyde solution. The whole blood was centrifuged at a speed of 3,000 rpm for 30 minutes, and the supernatant was separated and applied to the determination of cholesterol, low-density lipoprotein cholesterol (LDL-c), high-density lipoprotein cholesterol (HDL-c), and triglyceride by using correspondent kits (Nanjing Jiancheng Bioengineering Institute, China), or detection of glycosylated hemoglobin (HbA1c) and insulin by the ELISA detection kit (Abcam, USA). 


\section{H\&E examination and immunohistochemistry assay}

The tissues fixed in $4 \%$ formaldehyde were embedded in paraffin after dehydration in a gradient ethanol series $(70-100 \%)$. Cut embedded sections ( $4 \mu \mathrm{m}$ thick) with a rotary microtome and subjected to hematoxylin and eosin (H\&E) staining, Masson staining, periodic acid-Schiff (PAS) staining, and Sirius red staining, or labeled with TGF-ß1 (BD Biosciences Cat\# 562423, RRID: AB_2737615, Guangzhou, China) for microscopic examination. Sections were viewed with a light microscope (Olympus, Japan) and photographed at $\times 100$ magnification. The sections of histological examination and immunohistochemistry image were firstly labeled by an identification number code without the information of the grouping. The numbers of TGF- $\beta 1$ labelled cells in each slide were counted by using the Image J software (Macbiophotonics, Hamilton, Canada). Quantification analysis was performed in six randomly selected fields per sample in a blinded manner.

\section{Construction of HEK293-SGLT2 cell lines}

HEK293 cells (CLS Cat\# 300192/p777_HEK293, RRID: CVCL_0045) were seeded into a 6-well plate at the density of $0.5 \times 10^{6}$ cells/well and incubated overnight. To construct a cell line with over-expression of SGLT2, the plasmid plvx-puro-SGLT2 (containing SGLT2 gene sequence with lentivirus) were transfected into HEK293 cells using lipofectamine 3000 (Invitrogen, Cat: L3000-015, USA). After six-hour of infection, the culture medium was replaced with fresh Dulbecco's modified Eagle's medium (DMEM). After 48 hours, the cells were treated with puromycin (Thermo Fisher Scientific, USA, CID: 439530) at the final concentration of $2 \mu \mathrm{g} / \mathrm{mL}$, and the complete culture medium (with $2 \mu \mathrm{g} / \mathrm{mL}$ of puromycin) was replaced every 48 hours. RT-PCR and Western Blot were performed to determine the expression level of SGLT2.

\section{RNA extraction and RT-PCR}

Primer sequences were synthesized by Generay Biotech and the total RNA was extracted from cells using RNAiso Plus kit (Takara, Japan). The mRNA levels of SLC5A1, SLC5A2, and Actin were detected by the quantitative real time PCR. The primer sequences of SGLT1 and SGLT2 are as follows: SLC5A1F: GTCGGACTGTGGGCTATGTT, SLC5A1-R: GTCTGCAAGGTGTCCGTGTA; SLC5A2-F: ACGCCCATGTTTCTCATGGT, SLC5A2-R: CCTGGGGCTCATTCATCTCC.

\section{Western Blot}

The cells were washed 3 times with a $1 \times$ PBS buffer, then were lysed in an ice-colded RIPA extract buffer (FUDE, Cat: FD008, Jiangsu, China) supplemented with a mixture of protease inhibitors (SIGMA, Cat: \#021M8200, Guangzhou, China). The extracted proteins were separated by SDS-PAGE and transferred to a polyvinylidene difluoride (PVDF) membrane (Bio-Rad, Cat: \#1620177, USA). After blocking with a TBS/T buffer $(0.1 \%$, containing $5 \%$ bovine serum albumin) for $30 \mathrm{~min}$ at room temperature, the membrane was incubated with different primary antibodies at $4{ }^{\circ} \mathrm{C}$ for overnight, including SGLT2 antibody (Abcam, Cat\#ab37296), and Actin (Cell Signaling Technology Cat\# 4970). The membrane was washed with the TBS/T buffer four times to remove the unbound antibody and then incubated with the HRP-conjugated secondary antibody (Cell Signaling Technology). Protein bands were visualized with an ECL kit (Millipore, Cat: WBKLSO500). The densitometry analysis was performed using the Quantity One Software (Bio-Rad, CA, USA) relative to the loading control.

\section{2-NBDG uptake assay}

The cells were seeded into a 24-well glass bottom plate, and incubated at $37^{\circ} \mathrm{C}$ in a humidified incubator with $5 \% \mathrm{CO}_{2}$ until reached a density of $60 \%-70 \%$. Changed with a serum-free medium for two hours before taking experiment. After that, added $150 \mu \mathrm{L}$ sodium-HEPES buffer containing $240 \mu \mathrm{M} 2-\mathrm{NBDG}$ and 150 $\mu \mathrm{L}$ compound solutions to the plate to a final concentration of $10 \mu \mathrm{M}$. Incubated cells at $37^{\circ} \mathrm{C}$ for $30 \mathrm{~min}$. Then, washed cells with sodium-HEPES buffer three times and photographed with a Zeiss LSM laser confocal imaging system.

\section{Statistics analysis}


The data and statistical analyses were complied with the recommendations on experimental design and analysis in pharmacology (Curtis et al., 2018). Data were expressed as the mean \pm SEM. Data between two groups were analyzed by t-test using Graphpad Prism (Graphpad Software Inc, California, USA). Statistical analyses for multiple groups was performed by one-way ANOVA followed by Tukey's HSD post hoc tests. A $p$ value of [?] 0.05 was considered statistically significant.

\section{Nomenclature of targets and ligands}

Key protein targets and ligands in this article are hyperlinked to corresponding entries in http://www.guidetopharmacology.org the common portal for data from the IUPHAR/BPS Guide to PHARMACOLOGY(Harding et al., 2018), and are permanently archived in the Concise Guide to PHARMACOLOGY 2017/18(Alexander et al., 2017a; Alexander et al., 2017b).

\section{Results}

\section{CAG reduces plasma glucose in KM mice by inhibiting glucose uptake}

To determine the glucose-decreasing effect of AST andCAG (Chemical structure were listed in Figure 1A), KM mice were administrated with AST and CAG for ten days. Metformin and Dapagliflozin were used as positive drugs, respectively. Compared with the vehicle group, metformin and Dapagliflozin efficiently decreased plasma glucose at each time point, and Dapagliflozin showed a higher efficacy. AST could not show a significant effect on reducing plasma glucose, while CAG showed a more substantial hypoglycemic effect and accompanied by a backward shift of the blood glucose absorption peak (Figure 1B), indicating that CAG could decrease glucose absorption in mice. The area under the curve of plasma glucose (AUC) further confirmed the glucose-decreasing effect of CAG in mice, which was similar to metformin (Figure $1 \mathrm{C})$.

There are two approaches for glucose-diminishing in mice, including promoting the glucose uptake and mobilization or inhibiting glucose absorption (Pandey et al., 2015). To reveal the underlying possible pattern of the glucose-decreasing inCAG -treated mice, we performed glucose uptake assay in cells by using the 2( $N$-(7-nitrobenz-2-oxa-1,3-diazol-4-yl) amino)-2-deoxyglucose (2-NBDG) labelling method. Dapagliflozin markedly decreased glucose uptake by a decrease in the signal of the 2-NBDG within cells, and an opposite effect was observed in metformin-treated cells, which was in accordance with the previous studies (Lu et al., 2018). As shown in Figure 2, cells treated with CAG showed a similar pattern with that of Dapagliflozin, which was a significant reduction of the 2-NBDG signal. To be noted, metformin, Dapagliflozin, and CAG could all enhance insulin sensitivity as judged by an increase in the signal of the 2-NBDG in the combination group compared with groups in the absence of insulin. These results suggested that CAG, similar to Dapagliflozin, could inhibit glucose uptake.

\section{CAG ameliorates diabetes-related metabolic syndromes in ZDF diabetes rat}

ZDF diabetes rat, a well-known model mimicking the characteristics of T2DM populations, was applied to determine the anti-diabetes effects of CAG in vivo. The related indexes, including body weight, levels of glucose, HbA1c, triglyceride, cholesterol, LDL-c, and HDL-c in the ZDF rats treated with CAG for five weeks, were determined. As shown in Figure 3A, Dapagliflozin decreased plasma glucose after one week of treatment, and this strong glucose-decreasing effect did not maintain to 5 weeks as observed by a slight increase in plasma glucose level in week $2^{\sim} 5$. Compared with Dapagliflozin, CAG could reduce blood glucose levels with a concentration-dependent mode. Either the hypoglycemic effect or the later increase of glucose level is not as apparent as Dapagliflozin. The OGTT assay further confirmed that CAG had a mild hypoglycemic effect on plasma glucose in mice (Figure 3B and 3C).

In clinical, diabetes populations are accompanied by several metabolic syndromes (MetS), bodyweight decrease, and tissue breakdown, especially at the late stage. Thus, we evaluated the effect of CAG on bodyweight and observed a progressive decrease in body weight in the vehicle group, while Dapagliflozin and CAG efficiently reversed this decrease (Figure 3D). Consistent with the plasma glucose-decreasing effect in KM mice, CAG dose-dependently decreased serum glucose and HbA1c levels as well as the insulin excretion 
level, whereas Dapagliflozin showed no major effect on insulin level in rats (Figure 3E). Especially for another important clinical diagnosis index for diabetes, glycated hemoglobin (HbA1c)(Rodbard et al., 2007),CAG showed significant decreasing effect similar to Dapagliflozin (Figure 3E). Meanwhile, CAG could also efficiently decrease plasma triglyceride, low-density lipoprotein cholesterol (LDL-c) and reverse the progressive degeneration of ratio HDL-c/LDL-c in ZDF rats (Figure 3F-3I).

\section{CAG inhibits}

\section{coronary artery sclerosis, myocardial and kidney fibrosis in ZDF rats}

As mentioned above, complications are one of the main causes of death in patients with diabetes, and whether anti-diabetes drugs can alleviate these complications has become an important consideration in the development of such drugs. The main complication of diabetes is coronary artery sclerosis (Ikonomidis et al., 2016; Naito \& Miyauchi, 2017). Using H\&E and periodic acid-Schiff staining, we found that the CAG -treated mice had thinner coronary arteries than the vehicle group (Figure 4A and 4B). The thinning effect on the coronary arteries of CAG was even stronger than that of Dapagliflozin. This result corresponded to an increase in the plasma HDL-c/LDL-c ratio in rats treated withCAG (Figure 3I), suggesting that CAG can effectively reverse coronary atherosclerosis.

Also, epidemiological studies have shown that with the development of diabetes, multiple tissues collapse, such as myocardial fibrosis and renal degeneration, which can also lead to cardiovascular complications and diabetic nephropathy (Pop-Busui et al., 2017). We then determined the protective effects of CAG on cardiovascular and nephropathy complications in ZDF rats. By using the Sirius staining (for collagen) and Masson staining (for fiber), a significant accumulation of collagen in the heart, which is a pathologic indicator of myocardium fibrosis, was observed in the vehicle group (as indicated by the black arrow in Figure 5A), compared with the lean group. Dapagliflozin and CAG could efficiently decrease collagen and fiber to a similar level of that in the lean mice (Figure 5A-5C). These ameliorating effects were well coincided with their effects in reducing myocardial fibrosis in ZDF diabetes rats. These two compounds have also been shown to relieve fibrosis in the kidney similar to that in the heart. (Figure 5D-5F). Immunohistochemistry analysis of TGF- $\beta 1$ protein, a representative biomarker of fibrosis, further confirmed the anti-fibrosis effects of CAG in the heart and the kidney as indicated by a significant decrease in the expression of TGF- $\beta 1$ (Figure 5G-5H).

\section{CAG inhibits glucose reabsorption in ZDF rats}

Inhibiting glucose reabsorption in kidney and promoting glucose efflux out are using therapeutic approaches for diabetes in clinic. After the treatment of Dapagliflozin or CAG for one week, we tested the level of urinary glucose and sodium, and urine efflux volume in ZDF mice at different time points in 24 hours. Dapagliflozin significantly increased the urinary glucose and sodium in each period within a day, while showed nearly no effect on urine efflux volume (Figure 6A-6C). Similar to Dapagliflozin, CAG increased urinary glucose, urinary sodium but a marginally decrease in the urinary efflux volume (Figure 6A-6C). Similar results appeared in ZDF rats whose administration was extended to 5 weeks (Figure 6D-6F). Of note, after 5-week administration, bothCAG and Dapagliflozin decreased the water intake volume (Figure $6 \mathrm{G}$ ), suggesting the inhibitory effects on glucose reabsorption and the promotion of glucose efflux out.

\section{The anti-diabetic effect of CAG is related to the inhibition of SGLT2}

Considering that the antidiabetic effect and manner of CAG were similar to that of Dapagliflozin, we further studied the effect of CAG on SGLT protein. By using an immunohistochemistry detection, a higher expression of SGLT2 was observed in kidney of ZDF diabetic rats in the vehicle group compared with the lean mice, and a reversal effect of Dapagliflozin was also overserved, which was consistent with the previous reports. A similar decreasing was found in the CAG -treated diabetic rats (Figure 7A). Similarly,CAG and Dapagliflozin also inhibited the expression of SGLT2 in the HEK293 cell lines with over-expression of SGLT2 (Figure 7B and 7C).

\section{Discussion}


The present study investigated the therapeutic effects of CAG, the major metabolite of AST , for treating type 2 diabetes, and its underlying mechanism for the first time. AST is a glucoside isolated from traditional Chinese herb Astragalus Membranaceus (fisch) bunge, which is one of the most widely used traditional Chinese medicine for diabetes therapy (Wang et al., 2015). Till today, AST shows broadly pharmacological effects, such as reducing the symptoms of metabolic syndrome (Yue et al., 2017; Zhang et al., 2011), preventing the cardiovascular pathological changes and protecting against cardiovascular injury (Xu et al., 2006) and improving renal function (Lu et al., 2015), or reducing the progression of diabetic peripheral neuropathy (Yu et al., 2006). Despite these findings, the molecular mechanism by which AST fights diabetes remains elusive. In the present study, we tried to study the anti-diabetes mechanism of AST from another perspective, that is, to study the anti-diabetes effect and possible pathways of CAG , which is the aglycone of AST , as well as the major metabolic form of AST in vivo. The study of CAG not only helps to explain the antidiabetic action of AST in vivo but also helps to discover new active molecules of non-glycosides structure, which is undoubtedly meant for the research in this field. In fact, CAGshowed an improvement of pharmacokinetic profile over AST(Zhou et al., 2012), but whetherCAG had a promising anti-diabetes effect is not reported.

We examined the effect of CAG in the diabetic ZDF rat, which is a well-recognized model with characteristics towards diabetes as a manifestation of accompanied metabolic syndromes (MetS) in humans. Our results showed that CAG efficiently decreased plasma glucose, improved glucose tolerance, heart and kidney fibrosis, and coronary artery sclerosis. To be noted, the anti-diabetic effects of CAG shared a similar manner with Dapagliflozin rather than metformin, especially the manner in glucose uptake. Together with our further finding that CAG could down-regulated SGLT2's expression, CAG displays its anti-diabetes effects, at least in part, through the SGLT2 protein. These therapeutic effects suggest that CAG may be a considerable potential anti-diabetes candidate for further development.

SGLT2 is a novel plasma glucose regulator that responds for glucose (re)absorption by regulating glucose reabsorption in the kidney. SGLT2 is tissue-specific expressed in the kidney, and its expression level is inducible in response to the change of plasma glucose concentration (Chonlaket et al., 2018). Therefore, SGLT2 inhibitors are a new class of antidiabetic drugs with an insulin-independent mechanism. In particular, SGLT2 inhibitors reduce hospitalization for heart failure or cardiovascular mortality in patients with type 2 diabetes (Reid et al., 2020). We observed a reduction in the thickness of the artery wall in the mice-treated with CAG, together with a decrease of the expression of SGLT2 in cells and tissues. Moreover, CAG increased the urinary glucose, urinary sodium but decreased the urinary efflux volume, which are precisely the characteristics of SGLT2 inhibitors.

As talking above, there are lots of severe complications related to T2DM arising from chronic hyperglycemia. The majority of individuals with diabetes mainly die from cardiovascular disease. Recently, numerous trials have been carried out to ascertain the benefits of intensive glucose lowering on cardiovascular outcomes diabetes, such as using the SGLT2 inhibitors or combination therapy (Birkeland et al., 2017). The mechanism for SGLT2 inhibitor to reduce cardiovascular failure in T2DM patients includes reducing the oxygen consumption, improving the energy metabolism of the heart, and inhibiting the fibrosis of cardiomyocytes (Lam et al., 2016). Extensive evidence shows that there exists fibrosis in T2DM patients, including myocardial fibrosis (Guido et al., 2017) and kidney fibrosis (Tang et al., 2017). Diabetes-associated fibrosis is mainly mediated by activated fibroblasts, but may also involve fibrogenic actions of macrophages, fibroblasts, and vascular cells (Monami et al., 2017). To our delight, CAG showed an excellent effect in reversing myocardial fibrosis and renal fibrosis, although it was not as significant as Dapagliflozin in lowering blood glucose. To be specific, we found CAG significantly decreased the fiber contents in the tissues of the heart and kidney, accompanied by a significant reduction in the expression of TGF- $\beta$, which is the primary transcription activator involved in the regulation of fibrosis.

In conclusion, the present study identified the treating effects of CAG on both metabolic factors and diabetesrelated comprehensions. These data showed that CAG treatment significantly reduced plasma glucose and improved hyperinsulinemia in ZDF diabetic rats. CAG prevents cardio and renal dysfunction by inhibiting 
the accumulation of fibrosis in the heart and kidney of ZDF rats. Be noted, these treating effects of CAG were in line with an inhibition of plasma reabsorption in kidneys with SGLT2 as a key effector (Figure 8). Also, this study further provided new clues regarding the role of the SGLT2 inhibitor Dapagliflozin in treating diabetes and its related complications, such as cardiovascular disease and kidney dysfunction. Overall, these findings may provide a new insight into the novel application of CAG in the prevention of diabetes as well as the related metabolic comprehensions.

\section{Acknowledgement}

This work was supported by National Natural Science Foundation of China (81703336 to YR, 81673286 and 81922063 to TMO); the Ministry of Education of China (IRT-17R111); Guangdong Provincial Key Laboratory of Construction Foundation (2017B030314030).

\section{Conflict of interest}

This study does not incur financial or any other conflicts of interest.

\section{Author contribution}

This study was designed by T.-M. Ou, Y. Rao, and Q. Zhang. Q. Zhang, S. Q. Chen, J. W. Sun., Z. Y. Sun, W. Peng, G. T. Kuang, X. X. Su, X. N. Wang, performed biologic evaluation, animal study and mechanism study of AST and CAG . Y. Rao, T.-M. Ou provided reagents, materials and analysis tools. Data was analyzed and interpreted by Y. Rao, T.-M. Ou. The manuscript was written by Q. Zhang, Y. Rao. T.-M. $\mathrm{Ou}$, with input from all authors.

\section{Declaration of transparency and scientific rigour}

This Declaration acknowledges that this paper adheres to the principles for transparent reporting and scientific rigour of preclinical research as stated in the BJP guidelines for Immunoblotting and Immunohistochemistry, Animal Experimentation, and Design 83 Analysis and as recommended by funding agencies, publishers and other organizations engaged with supporting research.

\section{References}

Abdul-Ghani, M.A., Norton, L., DeFronzo, R.A. (2012). Efficacy and Safety of SGLT2 Inhibitors in the Treatment of Type 2 Diabetes Mellitus. Current Diabetes Report, 12: 230-238.

Alexander, S.P., Fabbro, D., Kelly, E., Marrion, N.V., Peters, J.A., Faccenda, E., et al. (2017a). THE CONCISE GUIDE TO PHARMACOLOGY 2017/18: Enzymes. British Journal Pharmacology, 174(Suppl 1): S272-S359.

Alexander, S.P., Peters, J.A., Kelly, E., Marrion, N.V., Faccenda, E., Harding, S.D., et al. (2017b). THE CONCISE GUIDE TO PHARMACOLOGY 2017/18: Ligand-gated ion channels.British Journal Pharmacology , 174(Suppl 1): S130-S159.

Birkeland, K.I., Jørgensen, M.E., Carstensen, B., Persson, F., Gulseth, H.L., Thuresson, M., et al.(2017). Cardiovascular mortality and morbidity in patients with type 2 diabetes following initiation of sodiumglucose co-transporter-2 inhibitors versus other glucose-lowering drugs (CVD-REAL Nordic): a multinational observational analysis. The Lancet Diabetes \&3 Endocrinology, 5: 709-717.

Chao, E.C., Henry R.R. (2010). SGLT2 inhibition - a novel strategy for diabetes treatment. Nature Reviews Drug Discovery, 9: 551-559.

Cheng, X.D., Wei, M.G. (2014). Profiling the metabolism of astragaloside IV by ultra performance liquid chromatography coupled with quadrupole/time-of-flight mass spectrometry.Molecules , 19: 18881-18896.

Chonlaket, P., Wongwan, T., Soodvilai, S. (2018). Liver X receptor activation inhibits SGLT2-mediated glucose transport in human renal proximal tubular cells. Experimental physiology, 103: 250-260. 
Dow, C., Mancini, F., Rajaobelina, K., Boutron-Ruault, M.C., Balkau, B., Bonnet, F., et al. (2018). Diet and risk of diabetic retinopathy: a systematic review. European journal of epidemiology, 33: 141-156.

Filippas-Ntekouan, S., Filippatos, T.D., Elisaf, M.S. (2018). SGLT2 inhibitors: are they safe? Postgrad Med , 130: $72-82$.

Gerstein, H.C., Miller, M.E., Byington, R.P., Goff, D.C., Bigger, J.T., Buse, J.B., et al.(2008). Effects of intensive glucose lowering in type 2 diabetes. The New England journal of medicine, 358: 2545-2559.

Guido, M.C., Marques, A.F., Tavares, E.R., Tavares de Melo, M.D., Salemi, V.M.C., Maranhao, R.C. (2017). The Effects of Diabetes Induction on the Rat Heart: Differences in Oxidative Stress, Inflammatory Cells, and Fibrosis between Subendocardial and Interstitial Myocardial Areas. Oxidative medicine and cellular longevity, 2017: 5343972.

Han, E., Jang, S.Y., Kim, G, Lee, Y.H., Choe, E.Y., Nam, C.M., et al. (2016). Rosiglitazone Use and the Risk of Bladder Cancer in Patients With Type 2 Diabetes.Medicine, 95: e2786.

Harding, S.D., Sharman, J.L., Faccenda, E., Southan, C., Pawson, A.J., Ireland, S., et al.(2018). The IUPHAR/BPS Guide to PHARMACOLOGY in 2018: updates and expansion to encompass the new guide to IMMUNOPHARMACOLOGY.Nucleic acids research, 46: D1091-D1106.

Hostalek, U., Gwilt, M., \& Hildemann, S. (2015). Therapeutic Use of Metformin in Prediabetes and Diabetes Prevention. Drugs , 75: 1071-1094.

Ikonomidis, I., Pavlidis, G., Lambadiari, V., Kousathana, F., Triantafyllidi, H., Varoudi, M., et al. (2016). Improvement of arterial stiffness and LV myocardial deformation in patients with coronary artery disease and diabetes mellitus type 2 after 6-month treatment with metformin and agonists of GLP-1R. European Heart Journal , 37: 176-177.

Lam, K.S., Chow, C.C., Tan, K.C., Ma, R.C., Kong, A.P., Tong, P.C., et al. (2016). Practical considerations for the use of sodium-glucose co-transporter type 2 inhibitors in treating hyperglycemia in type 2 diabetes. Current Medical Research Opinion, 32: 1097-1108.

Liu, X., Wang, W., Song, G., Wei, X., Zeng, Y., Han, P., et al. (2017). Astragaloside IV ameliorates diabetic nephropathy by modulating the mitochondrial quality control network. PloS one, 12: e0182558.

Liu, Y., Liu, W., Li, J., Tang, S., Wang, M., Huang, W., et al. (2019). A polysaccharide extracted from Astragalus membranaceus residue improves cognitive dysfunction by altering gut microbiota in diabetic mice. Carbohydrate Polymers , 205: 500-512.

Lu, W.S., Li, S., Guo, W.W., Chen, L.L., Li, Y.S. (2015). Effects of Astragaloside IV on diabetic nephropathy in rats. Genetics and molecular research. Genetics Molecular Research , 14: 5427-5434.

Lu, Y.T., Ma, X.L., Xu, Y.H., Hu, J., Wang, F., Qin, W.Y., et al. (2018). A Fluorescent Glucose Transport Assay for Screening SGLT2 Inhibitors in Endogenous SGLT2-Expressing HK-2 Cells. Natural Product Bioprospect, 9: 13-21.

Monami, M., Dicembrini, I., Mannucci, E. (2017). Effects of SGLT-2 inhibitors on mortality and cardiovascular events: a comprehensive meta-analysis of randomized controlled trials.Acta diabetologica , 54: 19-36.

Moucheraud, C., Lenz, C., Latkovic, M., Wirtz, V.J. (2019). The costs of diabetes treatment in low- and middle-income countries: a systematic review. BMJ global health , 4: e001258.

Naito, R., Miyauchi, K. (2017). Coronary Artery Disease and Type 2 Diabetes Mellitus Current Treatment Strategies and Future Perspective. International Heart Journal , 58: 475-480.

Nauck, M.A. (2014). Update on developments with SGLT2 inhibitors in the management of type 2 diabetes.Drug design, development and therapy, 8: 1335-1380. 
Navarro-Perez, J., Orozco-Beltran, D., Gil-Guillen, V., Pallares, V., Valls, F., Fernandez, A. et al. (2018). Mortality and cardiovascular disease burden of uncontrolled diabetes in a registry-based cohort: the ESCARVALrisk study. BMC cardiovascular disorders , 18: 180.

Pandey, A., Chawla, S., Guchhait, P. (2015). Type-2 diabetes: Current understanding and future perspectives.IUBMB life, 67: 506-513.

Pernicova, I., Korbonits, M. (2014). Metformin — mode of action and clinical implications for diabetes and cancer. Nature Reviews Endocrinology, 10: 143.

Pop-Busui, R., Braffett, B.H., Zinman, B., Martin, C., White, N.H., Herman, W.H., et al. (2017). Cardiovascular Autonomic Neuropathy and Cardiovascular Outcomes in the Diabetes Control and Complications Trial/Epidemiology of Diabetes Interventions and Complications (DCCT/EDIC) Study. Diabetes Care , 40: 94-100.

Ran, R.X., Zhang, C.Z., Li, R.S., Chen, B.W., Zhang, W.H., Zhao, Z.Y., et al. (2016). Evaluation and Comparison of the Inhibition Effect of Astragaloside IV and Aglycone Cycloastragenol on Various UDPGlucuronosyltransferase (UGT) Isoforms.Molecules, 21.

Reid, J., Rana, K., Niman, S., Sheikh-Ali, M., Lewis, T., Choksi, R.R., et al. (2020). Sodium-Glucose Cotransporter-2 (SGLT-2) Inhibitors for Cardiovascular Disease Prevention. American Journal of Cardiovascular Drug , 1-11.

Ren, C., Liu, W., Li, J., Cao, Y., Xu, J., Lu, P. (2019). Physical activity and risk of diabetic retinopathy: a systematic review and meta-analysis. Acta diabetologica , 56(1): 823-837.

Rieg, T., Vallon, V. (2018). Development of SGLT1 and SGLT2 inhibitors. Diabetologia , 61: 2079-2086.

Rives, M.L., Javitch, J.A., Wickenden, A.D. (2017). Potentiating SLC transporter activity: Emerging drug discovery opportunities. Biochemcal Pharmacology , 135: 1-11.

Rodbard, H.W., Blonde, L., Braithwaite, S.S., Brett, E.M., Cobin, R.H., Handelsman, Y., et al. (2007). American Association of Clinical Endocrinologists medical guidelines for clinical practice for the management of diabetes mellitus. Endocrinology Practice, 13(Suppl 1): 1-68.

Scheen, A.J. (2017). Dapagliflozin and saxagliptin tablets for adults with type 2 diabetes. Expert review of clinical pharmacology, 10: 1303-1316.

Shan, G., Zhou, X.J., Xia, Y., Qian, H.J. (2016). Astragalus membranaceus ameliorates renal interstitial fibrosis by inhibiting tubular epithelial-mesenchymal transition in vivo and in vitro. Experimental Therapeutic Medicine, 11:1611-1616.

Shyangdan, D.S., Uthman, O.A., Waugh, N. (2016). SGLT-2 receptor inhibitors for treating patients with type 2 diabetes mellitus: a systematic review and network meta-analysis.BMJ open , 6: e009417.

Szabo, N.J. (2014). Dietary safety of cycloastragenol from Astragalus spp.: Subchronic toxicity and genotoxicity studies. Food Chemical Toxicology , 64:322-334.

Tang, F., Hao, Y., Zhang, X., Qin, J. (2017). Effect of echinacoside on kidney fibrosis by inhibition of TGFbeta1/Smads signaling pathway in the $\mathrm{db} / \mathrm{db}$ mice model of diabetic nephropathy. Drug design, development and therapy, 11:2813-2826.

Tao, S., Li, L., Li, L., Liu, Y., Ren, Q., Shi, M., et al. (2019). Understanding the gut-kidney axis among biopsy-proven diabetic nephropathy, type 2 diabetes mellitus and healthy controls: an analysis of the gut microbiota composition. Acta diabetologica , 56: 581-592.

Vaduganathan, M., Butler, J. (2019). SGLT-2 inhibitors in heart failure: a new therapeutic avenue.Nature Medicine, 25: 1653-1654. 
Vaidya, V., Gangan, N., Sheehan, J. (2015). Impact of cardiovascular complications among patients with Type 2 diabetes mellitus: a systematic review. Expert Review Pharmacoeconomics Outcomes, 15: 487-497.

Vivian, E.M. (2015). Dapagliflozin: a new sodium-glucose cotransporter 2 inhibitor for treatment of type 2 diabetes. American journal of health-system pharmacy , 72: 361-372.

Wang, X.X., Levi, J., Luo, Y., Myakala, K., Herman-Edelstein, M., Qiu, L., et al. (2017). SGLT2 Protein Expression Is Increased in Human Diabetic Nephropathy. The Journal of biological chemistry, 292: 53355348.

Wang, Z.S., Xiong, F., Xie, X.H., Chen, D., Pan, J.H., Cheng, L. (2015). Astragaloside IV attenuates proteinuria in streptozotocin-induced diabetic nephropathy via the inhibition of endoplasmic reticulum stress. BMC Nephrology ,16: 44.

Wood, I,S,, Trayhurn, P, (2003). Glucose transporters (GLUT and SGLT): expanded families of sugar transport proteins. British Journal Nutrition, 89: 3-9.

Wright, E.M., Loo, D.D.F., Hirayama, B.A. (2011). Biology of Human Sodium Glucose Transporters.Physiological Review, 91: 733-794.

Xu, M.E., Xiao, S.Z., Sun, Y.H., Ou-Yang, Y., Zheng, X.X. (2006). Effects of astragaloside IV on pathogenesis of metabolic syndrome in vitro. Acta Pharmacol Sin,27: 229-236.

Yu, J., Zhang, Y., Sun, S., Shen, J., Qiu, J., Yin, X., et al. (2006). Inhibitory effects of astragaloside IV on diabetic peripheral neuropathy in rats. Canadian journal of physiology and pharmacology, 84:579-587.

Yue, S.J., Liu, J., Feng, W.W., Zhang, F.L., Chen, J.X., Xin, L.T., et al. (2017). System PharmacologyBased Dissection of the Synergistic Mechanism of Huangqi and Huanglian for Diabetes Mellitus. Front Pharmacology, 8. 694 .

Zhang, J., Wu, C., Gao, L., Du, G., Qin, X. (2020). Astragaloside IV derived from Astragalus membranaceus: A research review on the pharmacological effects. Advanced Pharmacology , 87: 89-112.

Zhang, N., Wang, X.H., Mao, S.L., Zhao, F. (2011). Astragaloside IV improves metabolic syndrome and endothelium dysfunction in fructose-fed rats. Molecules , 16: 3896-3907.

Zhou, R.N, Song, Y.L., Ruan, J.Q., Wang, YT, Yan, R. (2012). Pharmacokinetic Evidence on the Contribution of Intestinal Bacterial Conversion to Beneficial Effects of Astragaloside IV, a Marker Compound of Astragali Radix, in Traditional Oral Use of the Herb. Drug Metabolism Pharmacokonet, 27:586-597.

Zhou, X., Sun, X., Gong, X., Yang, Y., Chen, C., Shan, G., et al. (2017). Astragaloside IV from Astragalus membranaceus ameliorates renal interstitial fibrosis by inhibiting inflammation via TLR4/NF-small ka, CyrillicB in vivo and in vitro. International Immunopharmacolology, 42: 18-24.

\section{Figures Legends}

Figure 1. Effects of AST and CAG on plasma glucose and glucose tolerance in KM mice. 18-22 g male KM mice were gavage administrated with AST (5 mg/kg) and CAG $(5 \mathrm{mg} / \mathrm{kg})$ by for ten days. Fasted mice overnight and performed the OGTT experiment by orally administrated glucose at a dosage of $2 \mathrm{~g} / \mathrm{kg}$, determined plasma glucose at the time point of $0,15,30,60$, and $120 \mathrm{~min}$. The curve and area under curve (AUC) were calculated by Origin 8 software. Dapagliflozin $(1 \mathrm{mg} / \mathrm{kg})$ and Metformin $(100 \mathrm{mg} / \mathrm{kg})$ were used as positive controls. (A) Chemical structures of AST andCAG . (B) The content of plasma glucose in the OGTT experiment. (C) The calculated AUC in the OGTT experiment. ${ }^{*} p<0.05$, compared with the lean group; ${ }^{*} p<0.05$, compared with the vehicle group. $\mathrm{N}=8$ mice/group.

Figure 2 . Effect of CAG on glucose uptake in cells. HEK293 cells with over-expression SGLT2 were treated with CAG $(10 \mu \mathrm{M})$, Dapagliflozin $(10 \mu \mathrm{M})$, and Metformin $(1 \mathrm{mM})$ alone or combined with insulin $(10 \mu \mathrm{g} / \mathrm{mL})$ for $1 \mathrm{~h}$, then cells were stained with 2-NBDG $(120 \mu \mathrm{M})$ and Hoechst $33342(1 \mu \mathrm{g} / \mathrm{mL})$ at 37 ${ }^{\circ} \mathrm{C}$ for $30 \mathrm{~min}$. Representative images were captured and fluorescence were quantified. Image magnification, 
$\times 100 .{ }^{*} p<0.05$, compared with the vehicle cells; ${ }^{*} p<0.05$, compared with the insulin-treated cells. $\mathrm{N}=5$ independent experiments.

Figure 3. Determination of the effects of CAG on ameliorating diabetes related MetS in ZDF diabetes rats. ZDF diabetes rats were treated with Dapagliflozin $(1 \mathrm{mg} / \mathrm{kg} / \mathrm{d})$ and CAG $(1 \mathrm{or} 5 \mathrm{mg} / \mathrm{kg} / \mathrm{d})$ for five weeks, respectively. Relative parameters were determined. (A) Change of plasma glucose level in ZDF diabetes rats; (B-C) OGTT test after one-week treatment of drugs; (D) Change of body weight of the ZDF rats; (E) Levels of glucose, HbA1c, and insulin in serum, respectively; (F-H) Levels of triglylceride, cholesterol, and cholesterol in serum, respectively; (I) Ratio of HDL-c/LDL-c in serum. ${ }^{*} p<0.05$ compared with lean group mice; ${ }^{*} p<0.05$ compared with vehicle group mice. $\mathrm{N}=8$ mice / group.

Figure 4. Determination of the protective effect of CAG on coronary artery in ZDF diabetic rats. Rats were treated with Dapagliflozin $(1 \mathrm{mg} / \mathrm{kg} / \mathrm{d})$ and CAG $(1 \mathrm{or} 5 \mathrm{mg} / \mathrm{kg} / \mathrm{d})$ for five weeks, the tissues coronary artery were collected and subjected to HE and PAS staining. (A) Representative HE and PAS staining image of coronary artery. Image magnification, x200. (B) Determination of the thickness of coronary artery. ${ }^{\#} p$ $<0.05$, compared with lean group mice; ${ }^{*} p<0.05$, compared with vehicle group mice. $\mathrm{N}=8$ mice / group.

Figure 5. Determination of the anti-fibrosis effects of CAG in the heart and the kidney of ZDF diabetic rats. Rats were treated with Dapagliflozin $(1 \mathrm{mg} / \mathrm{kg} / \mathrm{d}$ ) and CAG (1 or $5 \mathrm{mg} / \mathrm{kg} / \mathrm{d}$ ) for five weeks, the tissues of heart and kidney were collected and subjected to pathological determination and immunohistochemistry assay for TGF- $\beta 1$. (A-C) Sirius red and Masson staining of the heart and quantification. Image magnification, $\times 200$. (D-F) Sirius red and Masson staining of the kidney and quantification. Image magnification, $\times 200$. (G-I) Determination of the protein TGF- $\beta 1$ in the heart and the kidney by using an immunohistochemistry assay and quantification. ${ }^{\#} p<0.05$, compared with lean group mice; ${ }^{*} p<0.05$, compared with vehicle group mice. $\mathrm{N}=8$ mice / group.

Figure 6. Effects of CAG on glucose reabsorption in the kidney of ZDF rats. Rats were treated with Dapagliflozin $(1 \mathrm{mg} / \mathrm{kg} / \mathrm{d}$ ) and CAG (1 or $5 \mathrm{mg} / \mathrm{kg} / \mathrm{d})$. After 1 week of treatment, the urinary glucose (A), urinary sodium (B) and urine efflux $(\mathrm{C})$ within 24 hours were determined. After 5 week of treatment, the total urinary glucose (D), urinary sodium (E) and urine efflux (F) in 24 hours were determined. (G) Effect of CAG on the water intake after 1 - or 5 -week treatment. ${ }^{*} p<0.05$, compared with lean group mice; ${ }^{*} p$ $<0.05$, compared with vehicle group mice. $\mathrm{N}=8$ mice / group.

Figure 7. Inhibition of CAG on SGLT2's expression. (A) Effects of CAG and Dapaglifloin on the expression of SGLT2 in the kidney of the ZDF rats by using an immunohistochemistry assay. (B) Determination of the SGLT2 expression in HEK293 cell by using Western blot. NC indicates cells without transfection by any plasmid, SGLT2 indicates cells transfected with the plasmid plvx-puro-SGLT2, and Ctrl indicates cells transfected with the control plasmid. (C) The HEK293 cells with over-expression of SGLT2 were treated with Dapagliflozin andCAG for 24 hours, and cells were subjected to SGLT2 determination by using Western blot.

Figure 8. Proposed mechanism underlying the anti-diabetes effects of CAG in ZDF diabetic rat. 
A

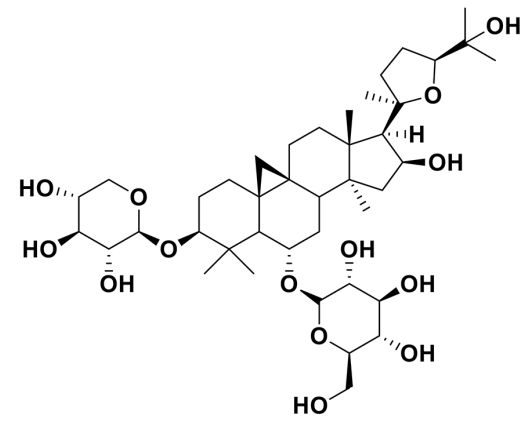

Astragaloside IV (AST)
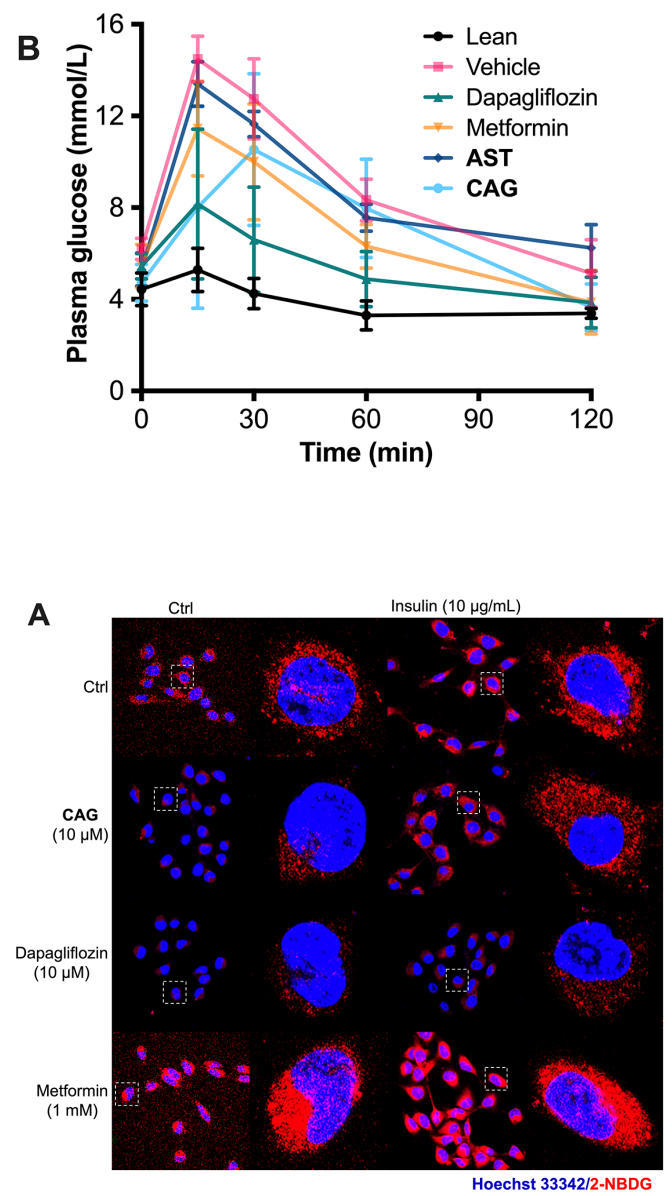

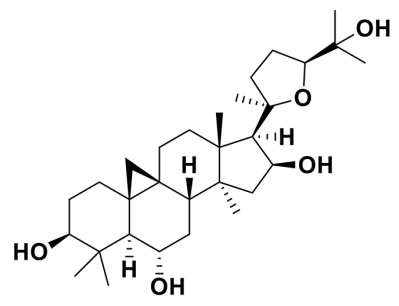

Cycloastragenol (CAG)
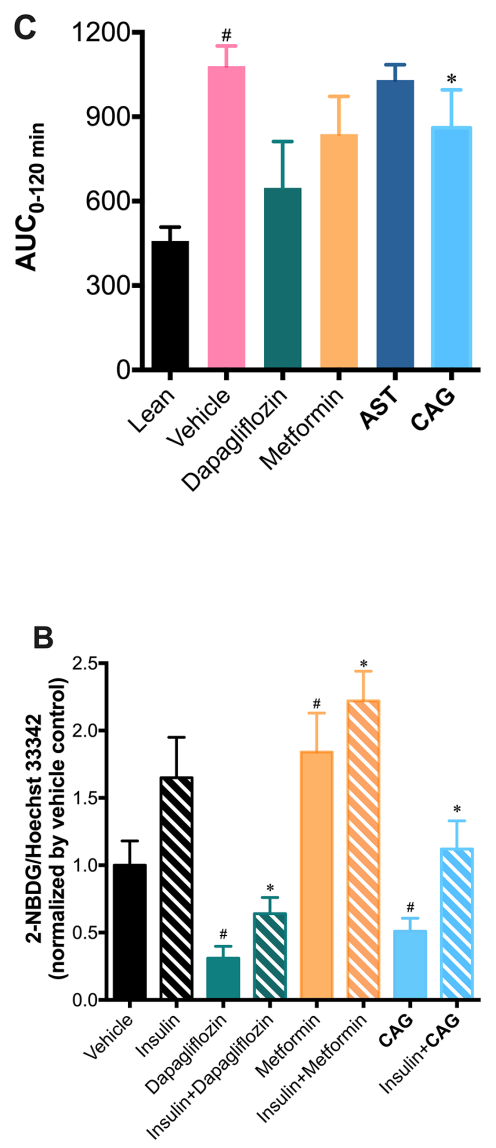

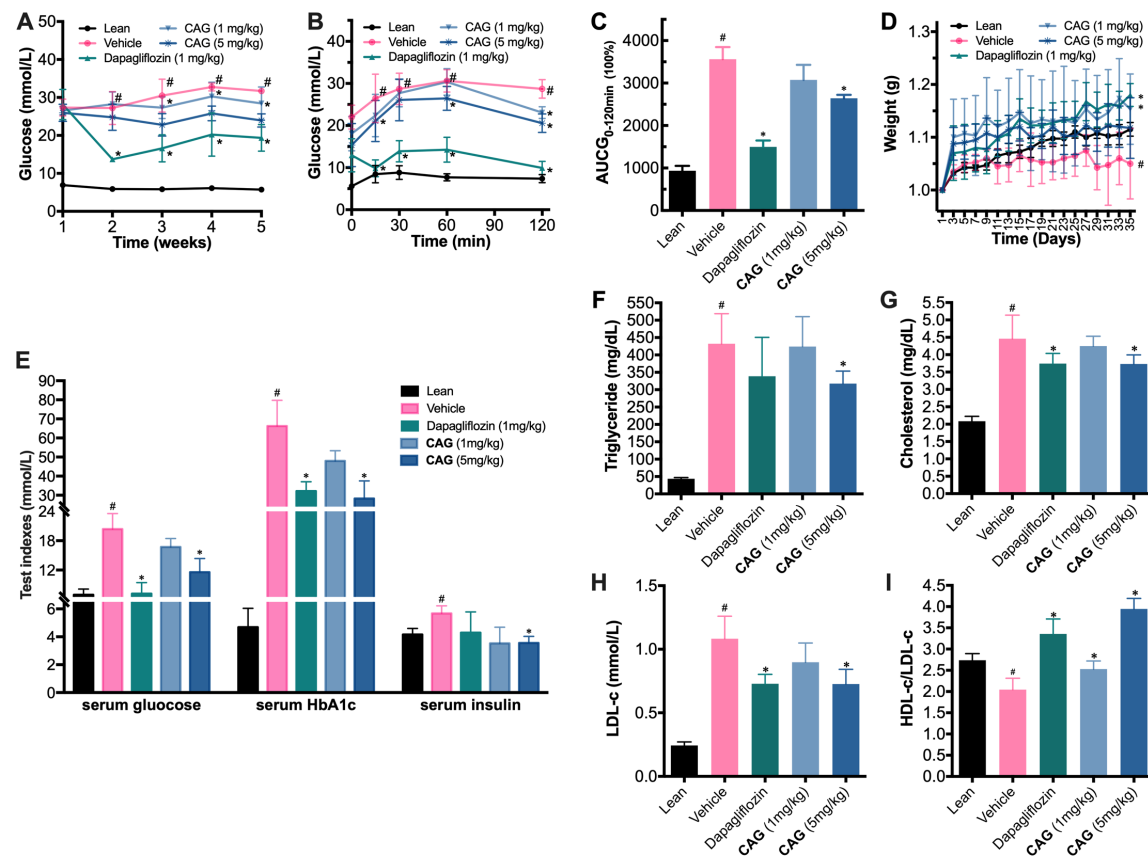

$G$

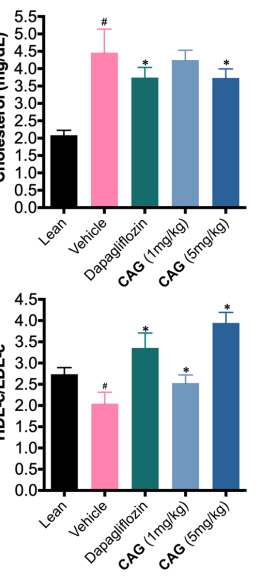

A

Coronary Artery
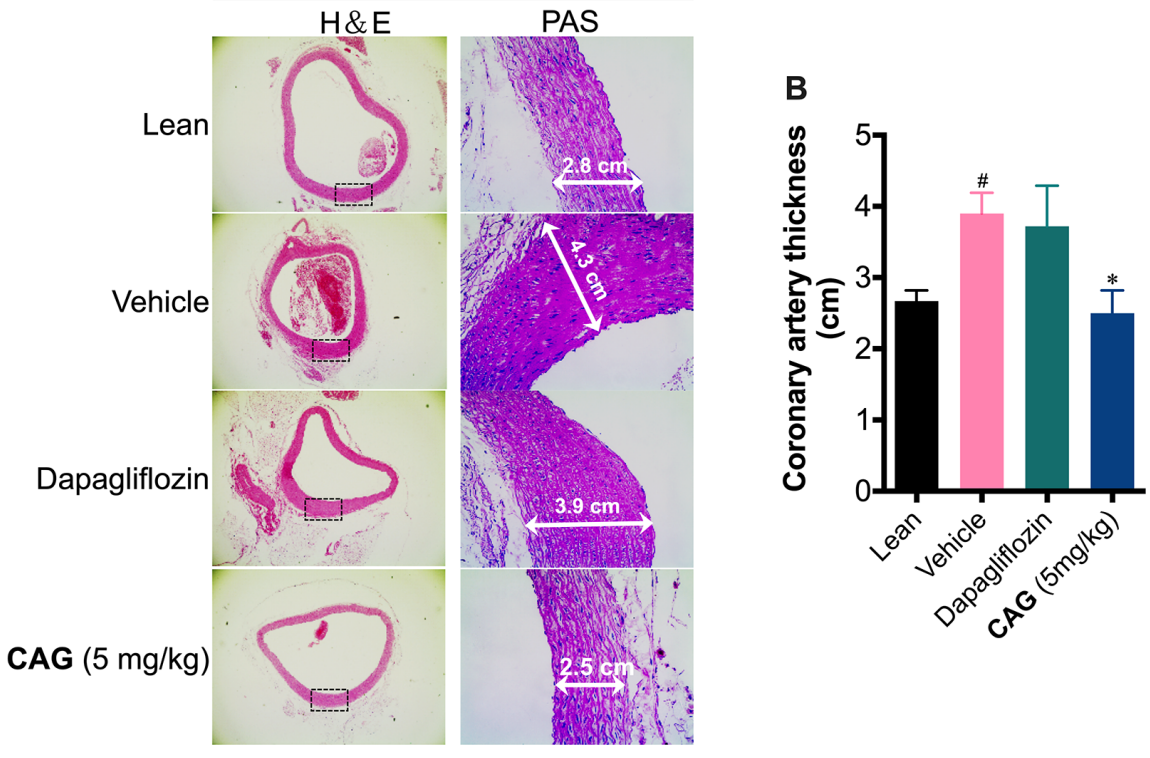

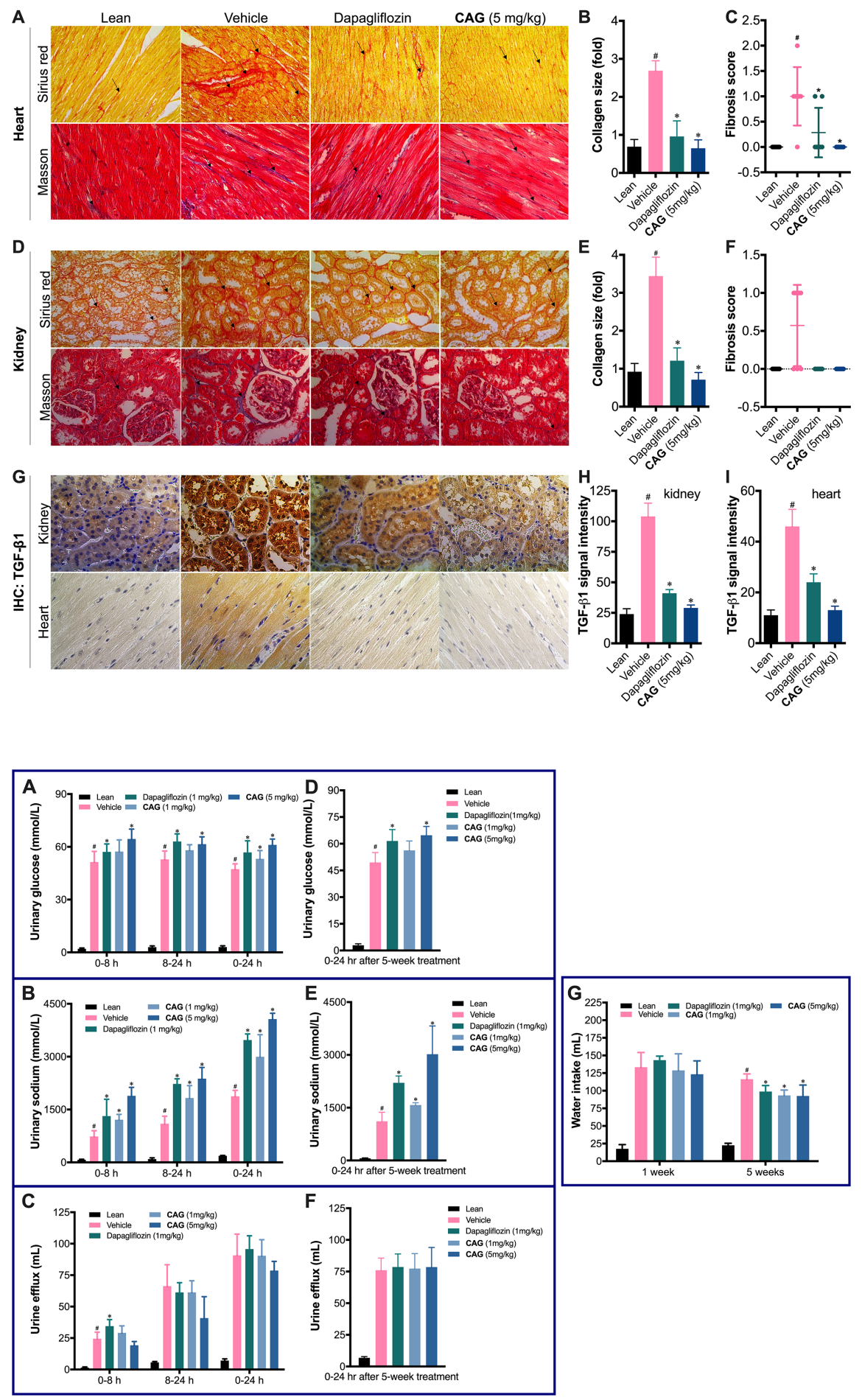
A

Vehicle Dapagliflozin CAG (1 mg/kg) CAG $(5 \mathrm{mg} / \mathrm{kg}$ )

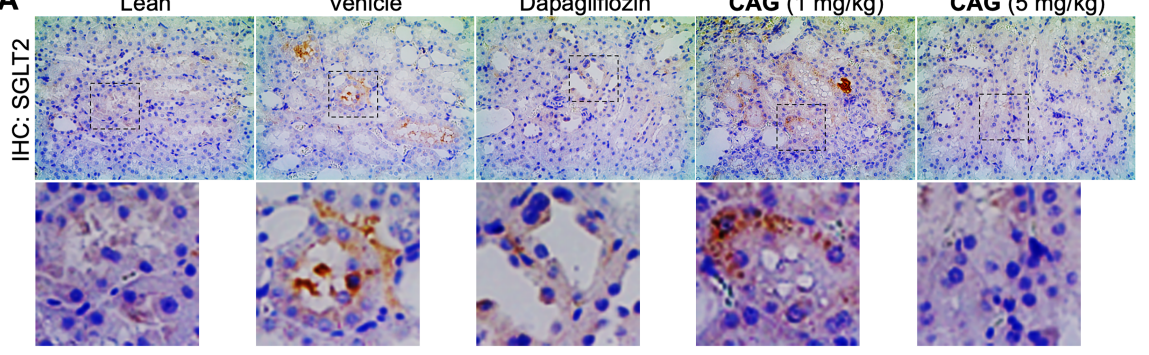
B

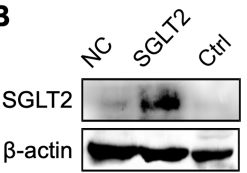

C

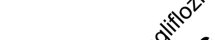

$c^{48} e^{10^{20}} c^{\circ 0^{\circ}}$

Urinary glucose

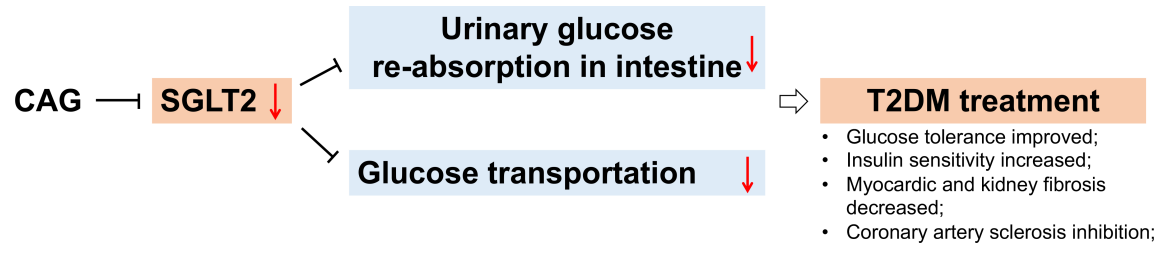

